Pacific Journal of Mathematic 


\title{
OPERATOR VALUED ROOTS OF ABELIAN ANALYTIC FUNCTIONS
}

\author{
Frank GilfeAther
}

In this paper, all spaces are separable Hilbert spaces and all operators are bounded linear transformations. Questions involving the structure of an operator for which an analytic function of it is normal or which satisfies a polynomial with certain operator coefficients have been considered and studied separately. Using von Neumann's reduction theory, a unified approach to these and similar questions can be given. This method yields generalizations of the cases which has been previously investigated, including structure results for $n$ normal operators. Through reduction theory of von Neumann algebras, the study of structural questions for a particular orerator is reduced to the properties of the often simpler, reduced operators. In all of the applications presented in this paper, the reduced operators will simply involve algebraic operators.

In $\S 1$, we introduce and study analytic functions $\psi(z)$, defined on a complex domain $\mathscr{D}$ and taking values in a commutative von Neumann algebra $\mathscr{A}$. Such a function will be called an abelian analytic function; and where there is any question, we shall specify the algebra $\mathscr{A}$. Using the direct integral decomposition of $\mathscr{A}$ into factors, we obtain the decomposition of $\psi$ into a normal family of scalar valued analytic functions on $\mathscr{D}$ indexed by a real variable. The main results in this section will be to show that the zeros of the scalar valued analytic functions can be chosen to be Borel functions of the real variable. We shall restrict our attention to a class of abelian analytic functions, called locally nonzero, so that each scalar valued analytic function in the corresponding normal family has no subdomain on which it is identically zero.

An operator $T$ in the commutant $\mathscr{A}^{\prime}$ of $\mathscr{A}$ is called a root of an abelian analytic function $\psi$, if $\sigma(T)$, the spectrum of $T$, is contained in $\mathscr{D}$ and $\psi(T)=0$ where $\psi(T)$ is to be defined in the usual $B^{*}$ algebraic manner or in an equivalent way using the direct integral decomposition of $\mathscr{A}$ into factors. Section 2 develops the structure for roots of locally nonzero abelian analytic functions. The main result, Theorem 2.1, states that the root of an abelian analytic function is "piecewise" a spectral operator of finite type. The structure theorem shows that roots of abelian analytic functions have hyperinvariant subspaces or are scalar multiples of the identity.

The remaining two sections of this paper are essentially appli- 
cations of the structure theorem for roots of abelian analytic functions to several classes of operators and the further use of reduction theory in their study. In $\S 3$, our investigation leads to theorems concerning solutions of

$$
f(A)=N,
$$

where $f$ is an analytic function on a domain containing $\sigma(A)$ and $N$ is a normal operator. The use of reduction theory in the study of (*) was introduced by the author in [9], and solutions of (*) have been previously studied by many authors with various restrictions on $f, A$, or $N$. The most complete investigation of the solutions of $\left(^{*}\right)$ has been done by C. Apostol in the setting of the theory of generalized spectral operators, however, his results are of a quite different nature from those given here [1]. If we set $\psi(z)=f(z)-N$, then ${ }^{\prime} s$ becomes an analytic abelian function and a solution $A$ of $\left({ }^{*}\right)$ is just a root of $\psi$. Hence, we may apply our methods and results; and in doing so, we are able to obtain two structure theorems for $A$. If there is no subdomain of on which $f$ is identically zero, then $f$ will be called locally nonzero. We show that whenever $A$ is a solution of $(*)$ where $f^{\prime}$ is locally nonzero and, of course, where $\sigma(A)$ is contained in $\mathscr{D}$, then it follows that $A$ is the direct sum of two operators; the first, $A_{1}$, which is algebraic and the second, $A_{2}$, which is "piecewise" similar to a normal operator. In the latter situation, the summand $A_{2}$ and the corresponding normal operator have the same spectrum. Under certain conditions, we may conclude that the solution $A$ of $(*)$ is "piecewise" similar to a normal solution $N_{0}$ of $\left({ }^{*}\right)$ and that $A$ and $N_{0}$ have the same spectrum. We also give a decomposition of certain operators satisfying $\left(^{*}\right)$ into direct summands each of which satisfy certain operator valued polynomials. Thus, we are able to generalize results obtained previously by C. Apostol, H. Radjavi, and P. Rosenthal and others [1, 10-13, 15, $16,18]$.

The structure of operators satisfying certain operator valued polynomials is studied in $\S 4$. An important class of such operators are the $n$-normal operators $(n \times n$ matrices of commuting normal operators). An $n$-normal operator $A$ satisfies a normal valued polynomial of degree $n$ by virtue of the Hamilton-Cayley Theorem; and moreover, the coefficients of the polynomial are in the center of the von Neumann algebra generated by $A$. N. Dunford has studied these operators primarily from the viewpoint of when they were spectral operators [6]. Since operators in a type $I_{n}$ von Neumann algebra are also $n$-normal, they naturally occur in the study of operator algebras. Also the structure and existence of hyperinvariant subspaces for certain $n$-normal operators have been investigated by 
various authors $[3-5,12,13,15]$. We may then apply the theorems in $\S 1$ to $n$-normal operators showing that they are "piecewise" similar to spectral operators and obtaining conditions for similarity which are compatible to those given in [6]. Whenever an operator $A$ satisfies a monic polynomial of degree less or equal to two with coefficients in the center of the von Neumann algebra generated by $A$, we can use reduction theory to obtain a complete structure theorem for it. This result will generalize results in $[3,16]$ and is closely connected to the work of A. Brown on binormal operators (2-normal) $[2,11]$.

Finally in $\S 4$, we give some sufficient conditions for a root of an abelian analytic function to be a spectral operator and, more specifically, a scalar type (similar to a normal operator) operator. For the n-normal case, our results complement those given by N. Dunford [6]. Also, we give some examples based on an example introduced by J. Stampfli of a 2-normal operator whose square is normal yet it is not similar to a normal square root of its square [18].

The essential component of von Neumann reduction theory is the concept of the direct integral decomposition of an algebra. For the details of the direct integral decomposition of a von Neumann algebra, we refer to [17]; however, we shall introduce some basic notations and results here. Let $\mu$ be the completion of a finite positive regular measure defined on the Borel sets of a separable metric space $\Lambda$, and let $e_{n}, 1 \leqq n \leqq \infty$ be a collection of disjoint Borel sets of $\Lambda$ with union 1. Let $H_{1} \subseteq H_{2} \subseteq \cdots \sqsubseteq H_{\infty}$ be a sequence of Hilbert spaces, with $H_{n}$ having dimension $n$ and $H_{\infty}$ being separable. By

$$
H=\int_{\Lambda} \oplus H(\lambda) \mu(d \lambda)
$$

we shall denote the space of weakly $\mu$-measurable functions from $\Lambda$ into $H_{\infty}$ such that $f(\lambda) \in H_{n}$, if $\lambda \in e_{n}$, and $\int_{\Lambda}\|f(\lambda)\|^{2} \mu(d \lambda)<\infty$. The space $H$ is a Hilbert space, and we shall denote the element $f \in H$ determined by the vector valued function $f(\lambda)$ as $\int_{A} \oplus f(\lambda) \mu(d \lambda)$.

An operator $A$ on $H$ is said to be decomposable if there exists a $\mu$-measurable operator valued function $A(\lambda)$ so that $(A f)(\lambda)=$ $A(\lambda) f(\lambda)$ for $f \in H$. The operator $A$ is denoted by

$$
A=\int_{\Lambda} \oplus A(\lambda) \mu(d \lambda) .
$$

Furthermore, every von Neumann algebra $\mathscr{A}$ on a separable space is spatially isomorphic to an algebra of decomposable operators on a direct integral of Hilbert spaces, such that the von Neumann algebra 
$\mathscr{A}(\lambda)$ generated by $\{A(\lambda)\}$, where $A \in \mathscr{A}$, is a factor $\mu$-a.e. Finally, we use the fact that if $A=\int_{1} \oplus A(\lambda) d \mu(\lambda)$ generates $\mathscr{A}$, then $A(\lambda)$ generates the von Neumann algebra $\mathscr{A}(\lambda) \mu$-a.e. Whenever in our use of this decomposition, there is no confusion over the space $A$, we shall suppress it.

If $A$ is an operator, we shall denote by $R(A), R(A)^{\prime}$, and $Z(A)$, respectively, the von Neumann algebra generated by $A$, the commutant of $R(A)$ and the center of $R(A)$. N. Suzuki has introduced the notion of a primary operator. One calls an operator $A$ primary, in case $R(A)$ is a factor; i.e., $Z(A)$ is just the scalar multiples of the identity. Let $A$ be defined on a separable Hilbert space and let $H=\int_{A} \oplus H(\lambda) \mu(d \lambda)$ be the direct integral decomposition of $H$ related to $R(A)$ for which the algebra $R(A)(\lambda)$ is a factor $\mu$-a.e., then this decomposition is unique in the sense of $[17 ;$ I. 6$]$. Thus, the operator $A$ is decomposed as $A=\int_{A} \oplus A(\lambda) \mu(d \lambda)$, where $A(\lambda)$ is primarily $\mu$-a.e., and we shall refer to this particular decomposition as the primary decomposition of $A$. We shall call a projection central for $T$ if it is in $Z(T)$. Finally, we shall let $R(z ; A)$ denote $(z I-A)^{-1}$.

1. Abelian analytic functions. In this section, we shall develop the notion of an abelian analytic function and investigate its properties. Let $\mathscr{A}$ be an abelian von Neumann algebra and $\psi(z)$, an $\mathscr{A}$ valued analytic function on a domain $\mathscr{D}$ in the complex plane, then $\psi$ is called an abelian analytic function with domain $\mathscr{D}$. For the usual facts about $B^{*}$ valued analytic functions, we refer to [7; III, 14].

Given an abelian von Neumann algebra $\mathscr{A}$, we may decompose it into a direct integral of factors. That is, $H$ is unitary equivalent to a direct integral of Hilbert spaces $\int_{A} \oplus H(\lambda) \mu(d \lambda)$, and this induces a spatial isomorphism between $\mathscr{A}$ and the diagonal operators on $\int_{A} \oplus H(\lambda) \mu(d \lambda)$. Thus, $H=\int_{A} \oplus H(\lambda) \mu(d \lambda)$; and for $A \in \mathscr{A}$, there is a unique $g \in L_{\infty}(\Lambda, \mu)$, so that $A=\int_{1} \bigoplus g(\lambda) I(\lambda) \mu(d \lambda)$, where $I(\lambda)$ is the identity operator on $H(\lambda)[17 ; 1,2.6]$.

Let $\psi$ be an abelian analytic function and $\mathscr{A}$ the corresponding von Neumann algebra with $\int_{A} \oplus H(\lambda) \mu(d \lambda)$ the decomposition of $H$ given above. Since $\psi(z)$ belongs to $\mathscr{A}$ for each $z$, we have

$$
\psi(z)=\int_{\Lambda} \oplus \psi(z, \lambda) I(\lambda) \mu(d \lambda)
$$

where $(\psi z, \lambda)$ corresponds via the isomorphism mentioned above to $\psi(z)$. We first give the relationship between the analyticity of $\psi(z)$ and that of $\psi(z, \lambda)$. 
Proposition 1.1. If $\psi(z)$ is an abelian analytic function with domain $\mathscr{D}$, then $\psi(z, \lambda)$, given by (1.1), is analytic on $\mathscr{D}$ for almost all $\lambda$ and $\|\psi(z, \lambda)\|_{\infty}$ is uniformly bounded on compact subsets of $\mathscr{D}$. Conversely, let $\psi(z, \lambda)$ be a family' of functions defined on $\mathscr{D} \times \Lambda$, where $\mathscr{D}$ is a complex domain. If $\psi(z, \lambda)$ is analytic in $z$ for almost all $\lambda$ on the domain $\mathscr{D}$ and if $\psi(z, \lambda) \in L_{\infty}(\Lambda, \mu)$ with $\|\psi(z, \cdot)\|_{\infty}$ uniformly bounded on compact subsets of $\mathscr{D}$, then $\psi(z)$, given by (1.1), is an abelian analytic function with domain $\mathscr{D}$.

Proof. We assume that $\psi$ is an abelian analytic function on $\mathscr{D}$ and that $z_{0} \in \mathscr{D}$. The series $\psi(z)=\sum N_{n}\left(\left(z-z_{0}\right)^{n} / n !\right)$ converges with $N_{n}$ given by Cauchy's formula is in $\mathscr{A}$ and $z$ is in some neighborhood $S_{0}$ of $z_{0}$. If $N_{n}=\int_{1} \oplus g_{n}(\lambda) I(\lambda) \mu(d \lambda)$, then for $z$ fixed in $S_{0}, \psi(z)(\lambda)=\sum_{n} g_{n}(\lambda)\left(\left(z-z_{0}\right)^{n} / n !\right) I(\lambda)$ for almost all $\lambda$. Hence, by the convergence properties of power series, we may conclude that $\psi(z, \lambda)$ is analytic in a neighborhood of $z_{0}$ and hence on $\mathscr{D} \mu$ a.e.

Conversely, we assume that $\psi(z, \lambda)$ belongs to $L_{\infty}(\Lambda, \mu)$ and $\|\psi(z, \cdot)\|_{\infty}$ is bounded for $z$ in compact subsets of $\mathscr{D}$. For $z_{0}$ in $\mathscr{D}$, let $\psi(z, \lambda)=\sum_{n} g_{n}(\lambda)\left(\left(z-z_{0}\right)^{n} / n !\right)$ be the power series expansion in a neighborhood $S_{0}$ of $z_{0}$. Since the functions $\left\{g_{n}\right\}$ are given by Cauchy's formula and $\psi(z, \cdot)$ is measurable, we conclude that $\left\{g_{n}\right\}$ are measurable. We are done if we can show that $g_{n} \in L_{\infty}(\Lambda, \mu)$. That, however, also follows from Cauchy's formula and using the hypothesis that $\|\psi(z, \cdot)\|_{\infty}$ are uniformly bounded on compact subsets of $\mathscr{D}$.

REMARK. If it is the case that $\psi(z, \lambda)$ is independent of $\lambda$, then the proposition is trivial. For example, if $\psi(z)=f(z) I$, then $\psi(z)(\lambda)=$ $f(z) I(\lambda)$ almost everywhere. In order to save the repetitiousness of deleting a set of measure zero from every argument, whenever $\psi(z)$ is an abelian analytic function on a domain $\mathscr{D}$, we will always assume that $\psi(z, \lambda)$ is analytic on a domain containing $\mathscr{D}$.

The main result in this section will show that the zeros of $\psi(z, \lambda)$ can be chosen in a $\mu$ measurable way. Such a result constitutes a generalization of the key lemmas in the study of $n$-normal operators by $\mathrm{N}$. Dunford $[6 ; \mathrm{XV}, 10]$ and is also related to the Theorem 1 in [5].

For this problem to be well defined, we must make a restriction so that $\psi(z, \lambda)$ is not identically zero on some subdomain of $\mathscr{D}$. We shall call an abelian analytic function $\psi$ locally nonzero if for every convergent sequence $\left\{z_{n}\right\}$ in $\mathscr{D}$ with $z_{n} \rightarrow z_{0}$ in $\mathscr{D}$ then $\bigcap_{n} \mathscr{N}\left(\psi\left(z_{n}\right)\right)=$ $\{0\}(\mathscr{N}(A)$ denotes the nullspace of the operator $A)$. For scalar valued functions, this is the usual definition of locally nonzero. To see this, we just let $H$ be one dimensional, then $\psi(z)$ is just a scalar 
valued function and $\mathscr{N}\left(\psi\left(z_{n}\right)\right) \neq\{0\}$ means that $\psi\left(z_{n}\right)=0$. The following lemmas establish the relationship between $\psi(z)$ and $\psi(z, \lambda)$ with respect to this property.

Lemma 1.2. An abelian analytic function $\psi$ is locally nonzero if and only if $\psi(\cdot, \lambda)$ is locally nonzero for almost all $\lambda$.

Proof. First assume that $\psi$ is not locally nonzero. That is, there exists a nonzero $x \in H$ and a sequence $\left\{z_{n}\right\}$ in $\mathscr{D}$ converging to $z_{0}$ in $\mathscr{D}$, so that $\psi\left(z_{n}\right) x=0$. If $E_{1}=\{\lambda \in \Lambda \mid x(\lambda) \neq 0\}$ and $E_{2}=$ $\bigcup_{n}\left\{\lambda \mid \psi\left(z_{n}, \lambda\right) x(\lambda) \neq 0\right\}$, then $E=E_{1} \backslash E_{2}$ is a set of positive measure on which $\psi(\cdot, \lambda)$ is not locally nonzero.

Conversely, if $\psi(\cdot, \lambda)$ is not locally nonzero for $\lambda$ in a set $E$ of positive measure, then we can show that $\psi(z)$ is not locally nonzero. For this, we let $\psi(z, \lambda)$ be zero on the subdomain $\mathscr{D}_{\lambda}$ if $\lambda \in E$. Since the domain of analyticity of $\psi(z, \lambda)$ contains $\mathscr{D}$, each $\mathscr{D}_{\lambda}$ contains one of the subdomains of $\mathscr{D}$; and thus, there is a subset $F$ of $E$ with positive measure so that $\bigcap_{\lambda \in F} \mathscr{D}_{\lambda} \supset \mathscr{D}_{0}$, a subdomain of $\mathscr{D}$. Therefore, $\psi(z, \lambda)=0$ for $\lambda \in F$ and $z \in \mathscr{D}_{0}$. Let $z_{n} \rightarrow z_{0}$ in $\mathscr{D}_{0}$ and $x \in H$ so that $\{\lambda \mid x(\lambda) \neq 0\}=F$, then $x \in \bigcap \mathscr{N}\left(\psi\left(z_{n}\right)\right)$. This completes the proof of this lemma.

Let a locally nonzero abelian analytic function $\psi$ be decomposed as in (1.1). The following theorem shows that the zeros of the functions $\psi(\cdot, \lambda)$ restricted to a compact subset of $\mathscr{D}$ can be made measurable.

THEOREM 1.3. Let $\psi(z, \lambda)$ be given by (1.1) with domain $\mathscr{D} \times \Lambda$. If $D$ is a bounded subdomain of $\mathscr{D}$ with $\bar{D} \subset \mathscr{D}$, then there exist disjoint Borel sets $E_{i}, i=0,1, \cdots$ with the measure of $\Lambda \backslash \bigcup_{i=0}^{\infty} E_{i}$ zero and for $\lambda \in E_{j}$, the analytic function $\psi(\cdot, \lambda)$ has exactly $j$ zeros counted to their multiplicities in $D$. Moreover, there exist Borel functions $\left\{r_{i}\right\}_{i=1}^{\infty}$ so that if $\lambda \in E_{j}$, then $r_{i}(\lambda) 1 \leqq i \leqq j$ are those zeros.

Proof. Since the number of zeros of an analytic function inside a desk is given by an integral formula, it is easy to see that if $n(\lambda)$ denotes the number of zeros counted to multiplicity of $\psi(z, \lambda)$ contained in $D$, then $S_{k}=\{\lambda \mid n(\lambda) \geqq k\}$ is Borel subset of $\Lambda$. Hence, if we may set $E_{k}=S_{k} \backslash S_{k+1}$, then $E_{k}$ is a Borel set; and it follows that $\Lambda \backslash \bigcup_{i=0}^{\infty} E_{i}$ has measure zero. We shall fix $n$ and define $r_{i}$ on $E_{n}$; and this will be clearly sufficient to complete the proof.

Henceforth, we are assuming that $E_{n}=\Lambda, 1 \leqq n<\infty$, and, the mapping $\psi$ on $D \times \Lambda$ is a Borel measurable map from the product space into the complex numbers. The projection of $\{(z, \lambda) \mid \psi(z, \lambda)=0\}$ onto $\Lambda$ is $\Lambda$ (a.e.) and by the Principle of Measurable Choice one 
finds a Borel function $r_{1}: \Lambda \rightarrow D$ so that $\left(r_{1}(\lambda), \lambda\right)$ is in the null space of $\psi$, that is, $\psi\left(r_{1}(\lambda), \lambda\right)=0$ for all $\lambda \in \Lambda$ [17; I, 4.7]. Consider now the function $\psi(z, \lambda)\left(z-r_{1}(\lambda)\right)^{-1} \equiv \phi(z, \lambda)$. By judiciously applying Schwartz's lemma on the modulus of a complex valued function one can show that $\phi(z, \lambda)$ is uniformly bounded in $\lambda$ on compact subsets in $\mathscr{D}$. Thus by Proposition 1.1 we conclude that $\phi$ is again an abelian analytic function. Moreover, it is clear that $\phi(\cdot, \lambda)$ has $n-1$ zeros in $D$ counted to their multiplicity almost everywhere. The proposition now follows with repeated application of the above argument.

The motivation for introducing abelian analytic functions is to study the structure of certain of their operator roots; and in doing so, unify several previous investigations. Whenever $\psi(z)$ is a polynomial with commuting normal coefficients and $T$ is an operator commuting with those coefficients, then $\psi(T)$ has an obvious definition. The definition of $\psi(T)$ we shall now give will be compatable with this usual definition when $\psi$ is a polynomial.

Let $\psi$ be an abelian analytic function on a domain $\mathscr{D}$ with values in the von Neumann algebra $\mathscr{A}$. If $H=\int_{A} \oplus H(\lambda) \mu(d \lambda)$ is the direct integral decomposition of $H$ corresponding to the decomposition of $\mathscr{A}$ into factors; and if $T \in \mathscr{A}^{\prime}$, then $T$ is a decomposable operator. That is, $T$ is represented as $T=\int_{\Lambda} \oplus T(\lambda) \mu(d \lambda)$ where $T(\lambda)$ is an operator on $H_{\lambda}$. Now let $T \in \mathscr{A}^{\prime}$ and $\sigma(T) \subset \mathscr{D}$. Since $\sigma(T(\lambda)) \subset \sigma(T)$, almost everywhere, the operator $\psi(T(\lambda), \lambda)$ is well defined by the usual functional calculus $[7,11]$.

To complete the definition of $\psi(T)$, let $\Gamma$ be an admissible curve for $\psi(T)$ in $\mathscr{D}$. Thus $\psi(T(\lambda), \lambda)=(2 \pi i)^{-1} \int_{\Gamma} R(z ; T(\lambda)) \psi(z, \lambda) d z$ and $\psi(T(\lambda), \lambda)$ is clearly a measurable operator function. If we can show that it is essentially bounded, then we may define $\psi(T)$ to be the decomposable operator given by $\psi(T)(\lambda)=\psi(T(\lambda), \lambda)$. Now let $z_{n}$ be a dense set on $\Gamma$. Since almost everywhere $\left\|R\left(z_{n} ; T(\lambda)\right)\right\| \leqq$ $\left\|R\left(z_{n} ; T\right)\right\|$, we may eliminate a set $E$ of measure zero and have on the complement of $E,\|R(z ; T(\lambda))\| \leqq\|R(z ; T)\|$ for all $z \in \Gamma$. By Proposition 1.1, $\|\psi(z, \lambda)\|_{\infty} \leqq M<\infty$ for all $z$ on $\Gamma$ and thus $\|\psi(z, \lambda) R(z ; T(\lambda))\| \leqq M$ on the complement of a set of measure zero and for all $z \in \Gamma$. Hence if $k=(2 \pi i)^{-1} \int_{\Gamma}|d z|$, we have that $\|\psi(T(\lambda), \lambda)\| \leqq M k$, for almost all $\lambda$ and therefore $\psi(T)$ is a bounded operator on $H$ if it is the decomposable operator defined by $\psi(T)(\lambda)=$ $\psi(T(\lambda), \lambda)$. It is clear that $\psi(T) \in \mathscr{A}^{\prime}$ since $\psi(T(\lambda), \lambda) \in \mathscr{A}^{\prime}(\lambda)^{\prime}$ for each $\lambda$. We conclude our remarks on the definition of $\psi(T)$ be noting that we have actually shown that $\psi(T)$ satisfies the conditions of a Fubini type theorem. Alternately $\psi(T)$ may be defined by usual $B^{*}$ 
algebraic techniques as

$$
\psi(T)=(2 \pi i)^{-1} \int_{\Gamma} \psi(z) R(z ; T) d z
$$

where $\psi(z)$ is a $\mathscr{A}$ valued analytic function defined on a domain containing $\sigma(T)$ and with $T \in \mathscr{A}^{\prime}$ and the integral converging in the norm. We may conclude that

$$
\begin{aligned}
\psi(T) & =\int_{\Lambda} \oplus(2 \pi i)^{-1} \int_{\Gamma} \psi(z, \lambda) R(z ; T(\lambda)) d z \mu(d \lambda) \\
& =(2 \pi i)^{-1} \int_{\Gamma} \int_{\Lambda} \bigoplus \psi(z, \lambda) R(z ; T(\lambda)) \mu(d \lambda) d z,
\end{aligned}
$$

that is, $\psi(T)(\lambda)=\psi(T(\lambda), \lambda)$ almost everywhere.

In the two applications of this theory, we wish to pursue we note that $\psi(T)$ coincides with previously understood definitions. If $\psi(z)$ is the polynomial $\psi(z)=N_{n} z^{n}+\cdots+N_{1} z+N_{0}$, with coefficients $N_{i}$ in an abelian von Neumann algebra, then by (1.3) we see that $\psi(T)$ is just $N_{n} T^{n}+\cdots+N_{1} T+N_{0}$. On the other hand, if $\psi(z)$ is a scalar valued analytic function, then by (1.3) we have established that $\psi(T)$ is the usual operator determined by the standard functional calculus [7; VII]. Moreover, in this latter case, the fact that the definition above for $\psi(T)$ and the usual one given by contour integration are the same as a special case of Theorem 1 in [11].

2. Roots of abelian analytic functions. We shall call $T$ a root of the abelian analytic function $\psi$ if $\psi(T)=0$ where $\psi(T)$ was defined in $\S 1$. If $\psi$ has domain of analyticity $\mathscr{D}$ and takes values in the von Neumann algebra $\mathscr{A}$, then, by the definition of $\psi(T)$, we are assuming that $T \in \mathscr{A}^{\prime}$ and that $\sigma(T) \subset \mathscr{A}$. In this section, we give a structure theorem for all roots of an abelian analytic function and several applications.

We shall state and prove the main theorem after which we shall restate it using the language of spectral operators.

THEOREM 2.1. Let $\psi$ be a locally nonzero abelian analytic function on $\mathscr{D}$ taking values in the von Neumann algebra $\mathscr{A}$ and let $T$ be a root of $\psi$. There exists a normal operator $S$ in $\mathscr{A}^{\prime}$ and $a$ sequence of mutually orthogonal projections $\left\{P_{n}\right\}$ in $\mathscr{A}$ with $I=\Sigma P_{n}$ so that $T P_{n}$ is similar to $\left(S+L_{n}\right) P_{n}$, where $L_{n}$ is a nilpotent operator $S L_{n}=L_{n} S$ and both $L_{n}$ and the operator which induces the similarity are in $\mathscr{A}^{\prime}$.

Proof. In assuming that $T$ is a root of $\psi(z)$ we have that $T \in \mathscr{A}^{\prime}$. We shall give the structure of $T$ by first decomposing $T$ 
into a direct integral of operators via the direct integral of decomposition of $\mathscr{A}$ and then determining the structure of each reduced operator in the decomposition of $T$.

Let $H=\int_{A} \oplus H(\lambda) \mu(d \lambda)$ be the decomposition of $H$ corresponding to the primary decomposition of $\mathscr{A}$. Since $T \in \mathscr{A}^{\prime}$, we may decompose $T$ as $T=\int_{A} \oplus T(\lambda) \mu(d \lambda)$. Furthermore, by $(1.3)$ if $\psi(T)=0$, then almost everywhere $\psi(T(\lambda), \lambda)=0$, where $\psi(z, \lambda)$ is an analytic function in a neighborhood of $\sigma(T(\lambda))$. By Lemma 1.2, the analytic function $\psi(z, \lambda)$ is locally nonzero in $\mathscr{D}$. In fact, by Theorem 1.3, there are disjoint Borel sets $E_{i}, i=0,1, \cdots$, where $\Lambda \backslash \bigcup_{i=0}^{n \infty} E_{i}$ has measure zero, and Borel functions $r_{i}(\lambda), i=1, \cdots$, so that if $\lambda \in E_{k}$ then $r_{1}(\lambda), \cdots, r_{k}(\lambda)$ are the zeros of $\psi(z, \lambda)$ in $\sigma(T)$ counted to their multiplicities. Since $\left\{E_{i}\right\}$ determine mutually orthogonal projections in $\mathscr{A}$, we may assume without loss of generality that for almost all $\lambda$ in $\Lambda, \psi(z, \lambda)$ has $k$ roots in $\sigma(T)$ counted their multiplicities and since $\psi(A(\lambda), \lambda)=0$ a.e., that $\mu\left(E_{0}\right)=0$.

It follows from the measurability of $\left\{r_{i}(\lambda)\right\}_{i=1}^{k}$, that the distinct roots of $\psi(z, \lambda)$ as well as their multiplicities can be chosen measurably. Thus we let $z_{1}(\lambda), \cdots, z_{n}(\lambda)$ be the distinct roots of $\psi(z, \lambda)$ in $\sigma(T)$ for $\lambda$ in the Borel set $F_{n}=\{\lambda \mid \psi(z, \lambda)$ has $n$ distinct roots in $\sigma(T)\}$ and let the multiplicity of $z_{i}(\lambda)$ be $k_{i}(\lambda)$. Define $\delta(\lambda)=\min _{i \neq j} \mid z_{i}(\lambda)-$ $z_{j}(\lambda) \mid$, which is also a Borel function. For each $i$, we determine the algebraic projections

$$
E_{i}(\lambda)=(2 \pi i)^{-1} \int_{\Gamma_{i}} R(z ; T(\lambda)) d z,
$$

where $\Gamma_{i}$ is the circle centered at $z_{i}(\lambda)$ of radius $\delta(\lambda) / 2$. Since $T(\lambda)$ is an algebraic operator with $\sigma(T(\lambda)) \subset\left\{z_{i}(\lambda)\right\}_{i=1}^{k}$ we have

$$
T(\lambda) / E_{i}(\lambda) H(\lambda)=\left[z_{i}(\lambda) I(\lambda)+N_{i}(\lambda)\right] / E_{i}(\lambda) H(\lambda),
$$

where $N_{i}(\lambda)$ is nilpotent of order $k_{i}(\lambda)$. Setting

$$
R(\lambda)=\left(\sum_{i=1}^{n} E_{i}(\lambda) E_{i}(\lambda)^{*}\right)^{1 / 2}
$$

then $R(\lambda)$ is invertible on $H(\lambda), R(\lambda) E_{i}(\lambda) R(\lambda)^{-1}=P_{i}(\lambda)$ are mutually orthogonal self-adjoint projections with $I(\lambda)=\sum_{i=1}^{n} P_{i}(\lambda)$, for $\lambda \in F_{n}$ and

$$
R(\lambda) T(\lambda) R(\lambda)^{-1}=\sum_{i=1}^{n} z_{i}(\lambda) P_{i}(\lambda)+L(\lambda),
$$

where $L(\lambda)^{k}=0$ and $P_{i}(\lambda) L(\lambda)=L(\lambda) P_{i}(\lambda)$ for each $i$. The form (2.4) is what we desired as our structure theorem. The only drawback to 
integrating the expression (2.4) over $F_{n}$ and then taking direct sums is the boundedness of the projections $E_{i}(\lambda)$ (the boundedness of $R(\lambda)$ and $R(\lambda)^{-1}$ only depend on $n$ and the boundedness of the $\left.E_{i}(\lambda)\right)$.

It is not the case that the projections $E_{i}(\lambda)$ are in general bounded independent of $\lambda$ and thus the structure theorem is given in terms of "piecewise" similarity. Let

$$
G_{m}=\left\{\lambda \in F_{n} \mid\left\|E_{i}(\lambda)\right\| \leqq m, i=1,2, \cdots, n\right\},
$$

and $g_{m}(\lambda)$ the characteristic function of the Borel set $G_{m}$. Let $Q_{m}$ be the corresponding projections in given by

$$
Q_{m}=\int_{\Lambda} \oplus g_{m}(\lambda) I(\lambda) \mu(d \lambda)
$$

and set $H_{m}=Q_{m} H$ and $T_{m}=T / H_{m}$. Then $R(\lambda), R(\lambda)^{-1}$ and $L(\lambda)$ are uniformly bounded for $\lambda \in G_{m}$ and hence we may define

$$
\begin{aligned}
& R_{m}=\left(I-Q_{m}\right)+\int_{\Lambda} \oplus g_{m}(\lambda) R(\lambda) \mu(d \lambda), \\
& N_{m}=\int_{\Lambda} \bigoplus g_{m}(\lambda) N(\lambda) \mu(d \lambda)
\end{aligned}
$$

and

$$
S=\int_{A} \oplus\left(\sum z_{i}(\lambda) P_{i}(\lambda)\right) \mu(d \lambda),
$$

where the summation under the integral in $S$ is taken over the number of distinct roots of $\psi(z, \lambda)$ in $\sigma(T)$, for example, $n$ for $\lambda$ in $F_{n}$. Considering all the special conditions on the operators, we have

$$
R_{m} T R_{m}^{-1} / Q_{m} H=\left[S+R_{m} N_{m} R_{m}^{-1}\right] / Q_{m} H
$$

or if we set $L_{m}=R_{m} N_{m} R_{m}^{-1}$, then

$$
R_{m} T R_{m}^{-1} Q_{m}=\left(S+L_{m}\right) Q_{m} .
$$

Finally, it is clear that $S \in \mathscr{A}^{\prime}$ is a normal operator, $L_{m} \in \mathscr{A}^{\prime}$ and $S L_{m}=L_{m} S$.

REMARK. Recently, decomposable operators on a direct integral of Hilbert spaces have been investigated by E. A. Azoff [2]. He has shown that in general, the spectrum of a decomposable operator is measurable. The results in $\S 1$ and this section imply this result for roots of abelian analytic functions, so that Azoff's work is related to certain results in these sections.

The following proposition will give a connection between the spectrum of $T$ and that of the corresponding normal operator $S$. 
This will be useful in the next section where we discuss special abelian analytic functions.

Proposition 2.2. If $T$ and $S$ are as in Theorem 2.1, then the spectrum of $S$ intersects every connected component of $\sigma(T)$.

Proof. Let $\mathscr{D}_{1}$ be a subdomain of $\mathscr{D}$ containing a connected component of $\sigma(T)$ and let $\Gamma=\partial \mathscr{D}_{1}$ be an admissible curve which also is contained in $\mathscr{D}$. Let $E=(2 \pi i)^{-1} \int_{r^{\prime}} R(z ; T) d z$, then $E \in \mathscr{A}^{\prime}$ and $E=(2 \pi i)^{-1} \int_{\Lambda} \oplus \int_{\Gamma} R(z ; T(\lambda)) d z \mu(d \lambda)=\int_{\Lambda} \oplus E(\lambda) \mu(d \lambda)[11]$. Clearly if $\int_{\Gamma} R(z ; T(\lambda)) d(z)=0$ almost everywhere, then $E=0$. Thus there is a Borel set $F$ so that $E(\lambda) \neq 0$ for $\lambda \in F$ and $\mu(F) \neq 0$. Hence, the set $G=\left\{\lambda \in F \mid \sigma(T(\lambda)) \cap \mathscr{D}_{1} \neq \phi\right\}$ and consequently for some $i$ the set $G_{i}=\left\{\lambda \in F \mid r_{i}(\lambda) \cap \mathscr{D}_{1} \neq \phi\right\}$ has positive measure. Therefore, $\sigma(S) \cap \mathscr{D}_{1}$ contains the essential range of $z_{i}$ restricted to $G_{i}$.

REMARK 1. The operator $S$ in the theorem is also a root of $\psi(z)$ as well as each of the operators $S+L_{m}$. Later we shall see that in special cases where the nilpotent part does not appear, we will then have all roots "piecewise" similar to normal roots.

REMARK 2. The proof of the theorem can be used to construct the normal as well as the nonnormal roots of $\psi(z)$. Thus we establish the fact that certain abelian analytic functions have roots. This is related to work in [4] and [12].

As we stated before the theorem, we may put this result in the context of the theory of spectral operators on a Hilbert space $H$. Our result in this setting then reads: Let $T$ be a root of a locally nonzero abelian analytic function. There exists mutually orthogonal projections $P_{n}$ in $R(T)^{\prime}$ so that $I=\sum P_{n}$ and $T / P_{n} H$ is a spectral operator of finite type.

Before giving an application of this result, we wish to remark on the roots of abelian polynomial functions vis-a-vis abelian analytic functions. If $f$ is a locally nonzero complex valued analytic function defined on a domain containing $\sigma(T)$, then $f(T)=0$ implies $p(T)=0$ for some complex valued polynomial. An analogous result holds for the operator valued analytic functions.

Proposition 2.3. If $T$ is the root of an abelian analytic function with values in $\mathscr{A}$, then $T$ is the direct sum of roots of monic polynomials with coefficients in $\mathscr{A}$. 
Proof. This follows from the structure theorem if we let $p_{N}(z, \lambda)=$ $\Pi_{i=1}^{N}\left(z-r_{i}(\lambda)\right)$, on the set where $N$ is the number of roots of $\psi(z, \lambda)$ in $\sigma(T)$ counted to their multiplicities and the Borel functions $r_{i}(\lambda)$ are the functions given in Theorem 1.3. Thus by equation (2.1) it follows that $p_{N}\left(T_{N}\right)=0$ where $T_{N}$ is defined in the obvious way.

We might point out the importance that a root $T$ of $\psi(z)$ belong to $\mathscr{A}^{\prime}$ aside from the fact that the proof of Theorem 2.1 would otherwise fail. In case $T$ is not in $\mathscr{L}^{\prime}$ essentially nothing can be determined, at least along the lines of our results. Let $H$ be a Hilbert space with orthonormal basis $\left\{e_{n}\right\}, n=0, \pm 1, \pm 2, \cdots$. If $U$ is the bilateral shift of $H$ with respect to this basis and $V$ is the unilateral shift on $\left\{e_{n}\right\}, n=0,1,2, \cdots$, and 0 on $\left\{e_{n}\right\}, 0=-1,-2, \cdots$, then $V$ satisfies the abelian polynomial $z^{2}-U z=\psi(z)$.

As a corollary to our main theorem, we shall show that roots of abelian analytic functions have hyperinvariant subspaces or are multiples of the identity operator. We shall call a closed subspace $M$ in $H$ hyperinvariant for an unbounded operator $A$, if $\overline{M \cap \mathscr{D}(A)}=M$ ( $\mathscr{D}(A)$ is the domain of $A$ and will be taken to be dense), and $M$ is invariant under every bounded operator $B$ which commuted with $A$ in the following sense: $B^{-1} \mathscr{D}(A) \cap \mathscr{D}(A)$ is dense and $A B=B A$ on $B^{-1} \mathscr{D}(A) \cap \mathscr{D}(A)$.

Let $A$ be an unbounded operator with dense domain and $T$ be a bounded operator. We say $T$ is quasisimilar to $A$, if there exist bounded one-to-one operators $X$ and $Y$, with dense ranges, so that $X H \subset \mathscr{D}(A), A X=X T$, and $T Y=Y A$ on $\mathscr{D}(A)$. The following lemma extends to the unbounded case a useful tool for proving the existence of hyperinvariant subspaces.

LEMMA 2.4. Let $T$ be quasisimilar to an unbounded operator A. If $A$ has nontrivial hyperinvariant subspaces, then $T$ has nontrivial hyperinvariant subspaces.

Proof. The proof is similar to the usual proof for the bounded case [13; Theorem 2.1].

Combining this lemma and Theorem 2.1, we have the following result, the proof of which is straightforward and it omitted.

THEOREM 2.5. Let $T$ be a root of an abelian analytic function. If $T$ is not a multiple of the identity, then $T$ has nontrivial hyperinvariant subspaces.

3. Solutions to $f(T)$ normal. In this section we develop the 
structure of the operator roots $T$ of the equation

$$
f(T)=N,
$$

where $f(z)$ is a complex valued analytic function on a domain $\mathscr{D} \supset \sigma(T)$ and $N$ is a normal operator. Certain results are known as was mentioned in the introduction; in particular, (3.1) has been studied with various restrictions on $f$. If we set $\psi(z)=f(z)-N$, then $\psi$ is a locally nonzero abelian analytic function on a domain $\mathscr{D}$ if and only if $f^{\prime}$ is locally nonzero on $\mathscr{D}\left(f^{\prime}\right.$ is locally nonzero is also expressed as $f$ is locally nonconstant). Thus we may apply the results of the previous sections to solutions of equation (3.1) whenever $f$ is locally nonconstant. The von Neumann algebra generated by $\{\psi(z) \mid z \epsilon$ $\mathscr{D}\}$ is abelian and in fact, just $R(N)$, the von Neumann algebra generated by $N$ and $I$. Hence, if $T$ has spectrum in $\mathscr{D}$ and $f(T)=N$, then $T$ commutes with $N$, so by the Fuglede theorem $T \in R(N)^{\prime}$ and hence $T$ satisfies the condition in the hypothesis of Theorem 2.1. Moreover, matters are even made simpler in this section if when we apply our results we let $\mathscr{A}=Z(T)$ as then we are utilizing the primary decomposition for $T$. Thus in this section, unless otherwise stated, $\mathscr{A}=Z(T)$ where $T$ is a solution of (3.1).

To aid in our characterization, we shall use the notion of semisimilarity, which is motivated by the use of a related concept by $\mathrm{A}$. Feldzamen for spectral operators [9]. We call $A$ and $B$ semi-similar if there exists a sequence of mutually orthogonal self-adjoint projections $\left\{P_{i}\right\}$ commuting with $A$ and $B$ so that $I=\sum_{i} P_{i}$ and for each $i$, there exists an invertible operator $S_{i}$ on $P_{i} H$, so that $S_{i}^{-1} A S_{i}=$ $B \mid P_{i} H$. That is, there is a "complete" family of reducing subspaces for $A$ and $B$, so that $A$ is similar to $B$ on each of these subspaces. Let $A$ and $B$ be semi-similar as above. By considering first the operator $X=\Sigma_{i}\left\|S_{i}\right\|^{-1} S_{i} P_{i}$ on $H$ and then $Y=\Sigma_{i}\left\|S_{i}^{-1}\right\|{ }^{-1} S_{i}^{-1} P_{i}$, we have that $A X=A B$ and $Y A=B Y$, where $X$ and $Y$ are quasiaffinities [14]. Thus this notion of semi-similarity implies the notion of quasisimilarity which is used by various authors to describe certain operators.

THEOREM 3.1. Let $f$ be a locally nonconstant analytic function on a domain $\mathscr{D}$ and let $N$ be a normal operator. If $T$ is an operator with $\sigma(T) \subset \mathscr{D}$ and $f(T)=N$, then there is a central projection $P$ of $T$ so that

$$
T=T_{0} \oplus T_{1},
$$

where $T_{0}=T \mid P H$ and $T_{1}=T \mid(I-P) H, T_{0}$ is semi-similar to a normal operator $N_{0}, \sigma\left(N_{0}\right)=\sigma\left(T_{0}\right)$ and $N_{0}$ is a normal solution to 
$f(\cdot)=N \mid P H . \quad$ Finally, $T_{1}$ is an algebraic operator with $f\left(T_{1}\right)=0$.

Proof. Let $H=\int_{A} \oplus H(\lambda) \mu(d \lambda)$ be the decomposition of $H$ so that $T=\int_{A} \oplus T(\lambda) \mu(d \lambda)$ is the primary decomposition of $T$. Since $N \in Z(T), N=\int_{\Lambda} \bigoplus g(\lambda) I(\lambda) \mu(d \lambda)$, where $g \in L_{\infty}(\Lambda, \mu)$, and moreover $f(T(\lambda))=g(\lambda) I(\lambda)$ almost everywhere [11].

Let $\lambda \in E_{0}$ if and only if $f(z)-g(\lambda)$ has only zeros of multiplicity one in $\sigma(T)$. If we let $g_{0}$ be the characteristic function of the set $E_{0}$, $P=\int_{\Lambda} \oplus g_{0}(\lambda) I(\lambda) \mu(d \lambda)$, then $T_{0}=T / P$ is easily seen to be semi-similar to a normal operator $N_{0}=N / P$ using Theorem 2.1.

On the complement of $E_{0}$, the function $f(z)-g(\lambda)$ has as least one multiple root. Since $f^{\prime}$ is locally nonzero there are only a finite number of distinct zeros of $f^{\prime}$ in $\sigma(T)$. Let $z_{1}, \cdots, z_{k}$ be the zeros of $f^{\prime}$ in $\sigma(T)$. Now a multiple root of $f(z)-g(\lambda)$ must be one of the numbers $z_{1}, \cdots, z_{k}$. Let $F_{i}$ be the measurable set of $\lambda$ in $\Lambda$ for which $f(z)-g(\lambda)$ has the multiple root $z_{i}$. Then $E_{i}=F_{i}-\bigcup_{j<i} F_{j}$ are disjoint measurable sets so that $\Lambda=\bigcup_{0}^{k} E_{i}$. If $\lambda_{1}, \lambda_{2} \in E_{j}(j>0)$, then $f(z)-g\left(\lambda_{1}\right)$ and $f(z)-g\left(\lambda_{2}\right)$ both have the root $z_{i}$ and therefore, $g$ is constant on each $E_{j}(j>0)$. If $g(\lambda)=a_{i}$ on $E_{i}(i>0)$, then $T(\lambda)$ satisfies the equation $f(z)-a_{i}$ for $\lambda$ in $E_{i}$ and it follows that $T(\lambda)$ satisfies a complex polynomial $p_{i}(z)$ for $\lambda \in E_{i}(i>1)$. Thus if $P_{1}=$ $I-P_{0}$ and $T_{1}=T \mid P_{1} H, p\left(T_{1}\right)=0$ for $p=p_{1} \cdots p_{k}$.

From Theorem 2.1 it is clear that $\sigma\left(N_{0}\right) \subset \sigma\left(T_{0}\right)$, in fact, $z$ belongs to the essential range of $z_{i}(\lambda)$ given in (2.4) for some $i$ if and only if $z$ is in $\sigma(N)$ and such a $z$ is in $\sigma(T)$. Conversely, we shall show that $\sigma\left(N_{0}\right) \supset \sigma\left(T_{0}\right)$. Let $N / P_{0} H=N_{1}$, then we are considering $f\left(T_{0}\right)=N_{1}$ and $T_{0}$ is semi-similar to $N_{0}$. Let $z_{0} \in \sigma\left(T_{0}\right)$ and $\varepsilon>0$ be given. Denote by $S_{0}$ a ball of radius $r$ less than $\varepsilon$, centered at $z_{0}$ with $\bar{S}_{0} \subset \mathscr{D}$, and with $f(z)-f\left(z_{0}\right) \neq 0$ on $\bar{S}_{0}$ except for $z=z_{0}$. Let $f\left(z_{0}\right)=z_{1}$, then by the spectral mapping theorem $z_{1} \in \sigma\left(N_{1}\right)$ and by the local mapping theorem, there exists a neighborhood $S_{1}$ of $z_{1}$ and $S_{2}$ of $z_{0}$ contained in $S_{0}$, so that $f\left(\bar{S}_{2}\right)=\bar{S}_{1}$.

Let $E(\cdot)$ be the spectral measure for $N_{1}$, then $E\left(S_{1}\right)$ is not zero since $z_{1} \in \sigma\left(N_{1}\right)$. Also $E\left(S_{1}\right) \in Z\left(T_{0}\right)$ so we denote $T_{01}$ to be $T / E\left(S_{1}\right) H$ and similarly $N_{01}$ and $N_{11}$. Thus, $f\left(T_{01}\right)=N_{11}$ and $N_{01}$ is the normal operator semi-similar to $T_{01}$ given by Theorem 2.1. Since $\sigma\left(N_{11}\right) \subset \bar{S}_{1}$, by the spectral mapping and local mapping theorems we have that $S_{2}$ must contain a component of $\sigma\left(T_{01}\right)$. By Proposition 2.2 there is a $z_{2}$ in $\sigma\left(N_{01}\right) \subset \sigma\left(N_{0}\right)$ so that $\left|z_{2}-z_{0}\right|<\varepsilon$. Since $\varepsilon$ was arbitrary, we may conclude that $\sigma\left(T_{0}\right) \subset \sigma\left(N_{0}\right)$ and the proof is complete.

Whenever $f^{\prime}$ has no zeros on $\sigma(T)$ then a theorem of C. Apostol 
has shown that $T$ is similar to a normal solution of (3.1) [1]. A generalization of that result will be given in Proposition 4.5. If, however, $f^{\prime}$ has zeros but $\left(f^{\prime}\right)^{-1}(0) \cap \sigma_{p}(T)$ is empty, then the operator $T_{1}$ does not occur need to in the above theorem and we have the following corollary.

COROLLARY 3.2. Let $f$ be a locally nonzero analytic function on a domain $\mathscr{D}$ and let $N$ be a normal operator. If $T$ is an operator with $\sigma(T) \subset \mathscr{D}, f(T)=N$ and $\sigma_{p}(T) \cap\left(f^{\prime}\right)^{-1}(0)=\phi$, then there exists a normal operator $N_{0}$ with $\sigma\left(N_{0}\right)=\sigma(T), f\left(N_{0}\right)=N$ and $T$ is semi-similar to $N_{0}$.

Prior to C. Apostol's work, it was shown by J. Stampfli that whenever $A^{n}$ is normal and $A$ is invertible, then $A$ is similar to a normal operator [18]. It easily follows from Stampfli's result that whenever $0 \notin \sigma_{p}(A)$, then $A$ is semi-similar to an $n$th root of $N$. This result is also an application of the above corollary where, of course, $f(z)=z^{n}$.

REMARK. That $\sigma\left(T_{0}\right)=\sigma\left(N_{0}\right)$ in Theorem 3.1 also follows the result of C. Apostol, C. Foias, and I. Colojoara when we have first shown that $T_{0}$ and $N_{0}$ are quasisimilar. For the first author proves that solutions of (3.1) are generalized scalar operators and the later authors have shown that quasisimilarity between decomposable operators preserves the spectrum. Since decomposable operators possess hyperinvariant subspaces, it follows from C. Apostol's results that solutions to (3.1) have hyperinvariant subspaces. However, this fact is also immediate by applying Theorem 2.5 to solutions of (3.1).

The following theorem and corollary generalize existing theorems and are obtained by placing some condition on $f(z)$. We shall only briefly indicate their proofs.

THEOREM 3.3. Let T satisfy (3.1) and let $\left\{z_{i}\right\}_{i=1}^{k}$ be the zeros of $f^{\prime}(z)$ in $\sigma(T)$ with multiplicities $\left\{n_{i}\right\}_{i-1}^{k}$. Assume that for each $i$ there exists a neighborhood $N_{i}$ of $z_{i}$ so that there are at most $m$ elements in $N_{i} \cap \sigma(T) \cap f^{-1}(z)$ for each $z$ in $\sigma(N)$. Then there exists an orthogonal projection $P$ in $R(T)^{\prime}$ so that

$$
T=T_{0} \oplus T_{1},
$$

where $T_{1}=T / P H$ is algebraic and satisfies $p(z)=\prod_{i=1}^{k}\left(z-z_{i}\right)^{n_{i}}$ and $T_{0}$ is similar to an operator $S_{0}$ which satisfies a monic abelian polynomial of degree at most $m$.

Proof. The proof is similar to the proof of Theorem 3.1 in that $T_{1}$ is the same operator in each case. Here because of the restriction 
on the spectrum we divide $\sigma\left(T_{0}(\lambda)\right)$ into at most $k$ distinct pieces so that each contains at most $m$ points of $\sigma\left(T_{0}(\lambda)\right)$ and each is of multiplicity one. From such a decomposition the theorem will follow.

COROLLARY 3.4. Let $T^{n}$ be normal where $\sigma(T)$ lies in $m$ sectors of the plane, each of width at most $2 \pi / n$, then $T$ is similar to the direct sum of a nilpotent operator $T_{0}$ and an operator $T_{1}$, which satisfies a polynomial of degree $m$ with coefficients in the center of the von Neumann algebra generated by $T_{1}$.

4. Operators satisfying an abelian polynomial. In this section, we give several results in the study of opetators which satisfy

$$
p(A)=0,
$$

where $p(z)$ is a monic polynomial with coefficients which are commuting normal operators and $A$ commutes with the coefficients. In view of Proposition 2.3, this problem subsumes the study of roots of abelian analytic functions. First, we shall discuss in some detail the results obtained whenever the polynomial is of degree two, and give results related to Corollary 3.2. As mentioned in the introduction, N. Dunford has studied $n$-normal operators from the viwpoint of when they were spectral operators. We relate our work to those results and to later works of T. Hoover [13] and H. Radjavi and P. Rosenthal [15, 16]. For example, several authors have shown that whenever $A$ is $n$-normal, then $A$ is a scalar multiple of the identity operator or $A$ has nontrivial hyperinvariant subspaces. These results also follow from Theorem 2.5.

Recently, H. Radjavi and P. Rosenthal have given a characterization of operators satisfying certain polynomials of degree 2 . Specifically, they have studied solutions to $z^{2}+a z=N$, where $N$ is a normal operator [16]. The following theorem generalizes their results and a similar result of $\mathrm{H}$. Behncke [3].

THEOREM 4.1. Let $T$ be a root of $p(z)$ where the degree of $p$ is less than or equal to 2 and the coefficients of $p(z)$ are in $Z(T)$. Then there exists a central projection $P$ of $T$, so that

$$
T=T_{0} \oplus T_{1}
$$

where $T_{0}=T / P H$ and $T_{1}=T /(I-P) H, T_{0}$ is normal, $T_{1}$ is unitarily equivalent to an operator of the form

$$
\left[\begin{array}{ll}
B & C \\
0 & D
\end{array}\right]
$$


on $K \oplus K$, where $B, C, D$ are commuting normal operators on $K$. Moreover, $\sigma(B) \cup \sigma(D)=\sigma\left(T_{1}\right)$ and $C$ is positive definite.

The proof of Theorem 4.1 will follow from a direct integral reduction of $T$ and the next lemma. Recall that an operator is called primary if the von Neumann algebra it generates is a factor. The following lemma has a direct elementary proof. However, it does follow from A. Brown's nonelementary work [4] and we cite that as a proof.

Lemma 4.2. Let $A$ be a primary operator on $H(\operatorname{dim} H>2)$. If $A^{2}+b A+c=0$ for complex numbers $b$ and $c$, then $A$ is unitarily equivalent to

$$
\left[\begin{array}{ll}
\gamma I & \beta I \\
0 & \alpha I
\end{array}\right]
$$

on $K \oplus K$, where $\{\gamma, \alpha\}=\sigma(A)=\left\{1 / 2\left(-b \pm\left(b^{2}-4 c\right)^{1 / 2}\right)\right\}$ and $\beta=\left(\rho^{2}-\right.$ $\left.|\alpha-\gamma|^{2}\right)^{1 / 2}$, where $\rho=\|A-\alpha I\|$.

Proof of Theorem 4.1. Let $T=T_{0} \oplus T_{1}$ be the unique central decomposition of $T$ by projection $P$ so that $T_{0}$ is normal and $T_{1}$ is completely nonnormal. If $T$ satisfies $T^{2}+T N_{1}+N_{2}=0$, then $T_{1}^{2}+$ $T_{1} L_{1}+L_{2}=0$ where $L_{i}=N_{i} /(I-P) H$ and $L_{i} \in Z\left(T_{i}\right)(i=1,2)$. We decompose $H_{1}=(I-P) H$ by the primary decomposition of $T_{1}$. Thus $H_{1}=\int_{\Lambda} \oplus H(\lambda) \mu(d \lambda)$ and

$$
T_{1}=\int_{\Lambda} \oplus T_{1}(\lambda) \mu(d \lambda),
$$

where $T_{1}(\lambda)$ is a primary operator defined on $H_{\lambda}$. Moreover, there exist bounded Borel functions $f_{1}$ and $f_{2}$ on $\Lambda$ so that for $i=1,2$,

$$
L_{i}=\int_{\Lambda} \oplus f_{i}(\lambda) I(\lambda) \mu(d \lambda) \text {. }
$$

Therefore, we may conclude that

$$
T_{1}(\lambda)^{2}+f_{1}(\lambda) T_{1}(\lambda)+f_{2}(\lambda) I(\lambda)=0
$$

almost everywhere. From our proposition, $T_{k}(\lambda)$ is unitarily equivalent to

$$
\left[\begin{array}{ll}
g(\lambda) I_{\lambda} & h(\lambda) I_{\lambda} \\
0 & k(\lambda) I_{\lambda}
\end{array}\right]
$$

on $K_{\lambda} \oplus K_{2}$ where $I_{\lambda}$ is the identity operator on $K_{\lambda}$, where $g, h$, and 
$k$ are measurable, $h(\lambda)>0$ and the projection $P(\lambda)$ onto the subpace $K_{\lambda} \oplus 0$ is measurable. We let $Q(\lambda)=I(\lambda)-P(\lambda)$ and then $P(\lambda) T_{1}(\lambda) P(\lambda)=g(\lambda) P(\lambda), P(\lambda) T_{1}(\lambda) Q(\lambda)=0, P(\lambda) T_{1}(\lambda) Q(\lambda)=h(\lambda) P(\lambda)$ and $Q(\lambda) T_{1}(\lambda) Q(\lambda)=k(\lambda) Q(\lambda)$ and the result follows.

Remark 1. That $N_{1}, N_{2} \in Z(A)$ is not essential to Theorem 4.1. The same conclusion holds if $A$ is any root of a locally nonzero abelian polynomial of degree less or equal to 2 . We need only decompose $A$ as in Theorem 2.1 and thus have $g(\lambda) A(\lambda)^{2}+h(\lambda) A(\lambda)+k(\lambda) I(\lambda)=0$ almost everywhere. By Theorem 4.1, there exists a projection $Q(\lambda)$ measurable with respect to $\lambda$, so that $A(\lambda) Q(\lambda)=r_{1}(\lambda) Q(\lambda)$, $P(\lambda) A(\lambda) P(\lambda)=r_{2}(\lambda) Q(\lambda)$ where $P(\lambda)=I(\lambda)-Q(\lambda)$ and $Q(\lambda) A(\lambda) P(\lambda)=$ $c(\lambda) Q(\lambda)$ where $c(\lambda)$ is a positive operator on $H(\lambda)$. The more general result now follows.

REMARK 2. A. Brown called 2-normal operators binormal and $\mathrm{H}$. Gonsher called them $J_{2}$ operators $[4,12]$. Hence, Theorem 4.1 implies that: $A$ is a binormal operator if and only if $A$ is a zero of a locally nonzero abelian polynomial of degree less than or equal to 2. For a discussion of the unitary invariant of these operators we refer the reader to [2].

We can obtain various known theorems as special cases of the preceeding theorems. For example, we can generalize Theorem 3 in [16] with the following corollary.

CoRollary 4.3. Let $T^{n}=N$, where $N$ is normal and let $\sigma(T)$ lie in two sectors of the plane each with width less than $2 \pi n^{-1}$. Then there are mutually orthogonal central projections $P_{0}, P_{1}$, and $P_{2}$ of $T$ with $I=P_{0}+P_{1}+P_{2}$ and

$$
T=T_{0} \oplus T_{1} \oplus T_{2}
$$

where $T_{0}=T / P_{0} H$ is nilpotent of order $n, T_{1}=T / P_{1} H$ is normal and $T_{2}=T / P_{2} H$ is unitarily equivalent to

$$
\left[\begin{array}{ll}
B & C \\
0 & D
\end{array}\right],
$$

where $B, C$, and $D$ are commuting normal operators with $C$ positive definite.

Proof. Let $P_{0}$ be the central projection so that $T / P_{0} H$ is normal and $T /\left(I-P_{0}\right) H$ is completely nonnormal. If we apply Corollary 3.6 to $T /\left(I-P_{0}\right) H$ we can obtain $P_{1}$ and $P_{2}$ so that $T / P_{1} H$ is algebraic and in fact $T^{n} / P_{1} H=0$ and $T / P_{2} H$ satisfies a monic polynomial of 
degree 2 with coefficients in $Z\left(T / P_{2} H\right)$. Using Theorem 4.1 we now conclude the complete structure of $T$.

In Theorem 2.1 we see that if the root functions are different almost everywhere, then the operator zero is semi-similar to a normal zero. We use this observation in the following result concerning solutions of an abelian polynomial of degree 2 which will be useful. It differs from the preceding results in that it utilizes semi-similarity.

Proposition 4.4. Let T satisfy an abelian polynomial of degree 2. Then there exists unique central decomposition of $T$ into

$$
T_{0} \oplus T_{1}
$$

so that $T_{0}$ is unitarily equivalent to the commuting sum of a normal operator and a nilpotent operator of index 2 . The operator $T_{0}$ has no reducing subspace on which it is similar to a normal operator and $T_{1}$ is semi-similar to a normal operator.

Proof. We let the root functions be $\left\{r_{i}(\lambda)\right\}_{i=1}^{2}$ and set $M=$ $\left\{\lambda \mid r_{1}(\lambda)=r_{2}(\lambda)\right\}$. If $g$ is the characteristic function of $M$, then $P=\int_{A} \oplus \mathrm{g}(\lambda) I(\lambda) \mu(d \lambda)$ is a central projections for $T$. We let $T_{0}$ be the completely nonnormal part of $T / P$ and the proposition follows from the fact that on the complement of $M, r_{1}(\lambda) \neq r_{2}(\lambda)$ almost everywhere.

In the case of operators satisfying an abelian analytic function, we always have by Theorem 2.1 that they are piecewise similar to spectral operators. The question naturally arises as to when are they spectral. This has been studied by both N. Dunford and C. Apostol for the special cases they considered respectively $[1,6]$. The following sufficient condition follows easily from the proof of Theorem 2.1.

Proposition 4.5. Let $T$ be a root of a locally nonzero analytic abelian function $\psi$ which has root functions $\left\{r_{i}(\lambda)\right\}_{i=1}^{m}$ in $\sigma(T)$ satisfying $\Pi_{i \neq j}\left|r_{i}(\lambda)-r_{j}(\lambda)\right| \geqq \delta>0$ almost everywhere. Then $T$ is similar to a normal root of $\psi$.

Proof. The root functions are given by Theorem 1.3 and under the assumption $\Pi_{i \neq j}\left|r_{i}(\lambda)-r_{j}(\lambda)\right|>0$ almost everywhere we have no multiple roots. Furthermore, the projections given by equation (2.1) are just $E_{i}(\lambda)=p_{i}(T(\lambda))$ where $p_{i}(z)=\Pi_{j \neq i}\left(z-r_{j}(\lambda)\right)\left(r_{i}(\lambda)-\right.$ $\left.r_{j}(\lambda)\right)^{-1}$ and are essentially bounded under the hypothesis on $\left\{r_{i}(\lambda)\right\}$.

In fact, a necessary and sufficient condition can be given in case $\Pi_{i \neq j}\left(r_{i}(\lambda)-r_{j}(y)\right) \neq 0$ almost everywhere. 
Proposition 4.6. If $T$ is a solution of an abelian analytic function with $\prod_{i \neq j}\left(r_{i}(\lambda)-r_{j}(\lambda)\right) \neq 0$ almost everywhere, then $T$ is a scalar type operator if and only if $\Pi_{i \neq j_{0}}\left(r_{r_{0}}(\lambda)-r_{2}(\lambda)\right)^{-1} \| T(\lambda)-$ $r_{\imath}(\lambda) \|$ is essentially bounded for $1 \leqq i_{0} \leqq n$.

REmark. The theorem of J. Stampfli for $T^{n}$ normal and $T$ invertible as well as S. Foguel's theorem and C. Apostol's theorem for $p(T)$ normal and $p^{\prime}(z) \neq 0$ on $\sigma(T)$ and $f(T)$ normal and $f^{\prime}(z) \neq 0$ on $\sigma(T)$ respectively, follow from these propositions.

Unfortunately, these conditions are not sufficient as we shall see below. In the case of an operator $T$ satisfying a second degree monic polynomial with coefficients in $Z(T)$, we can given necessary and sufficient for that $T$ be similar to a normal solution of the polynomial.

THEOREM 4.7. Let T satisfy a monic second degree polynomial with coefficients in $Z(T)$. If $T=\int_{\Lambda} \oplus T(\lambda) \mu(d \lambda)$ is the primary decomposition of $T,\left\{r_{i}(\lambda)\right\}_{i=1}^{2}$ are the root functions of the polynomial and $\rho(\lambda)=\left\|T(\lambda)-r_{1}(\lambda)\right\|$, then $T$ is a spectral type operator of nilpotent index 2 if and only if $\left\{\rho(\lambda)\left|r_{1}(\lambda)-r_{2}(\lambda)\right|^{-1}: r_{1}(\lambda) \neq r_{2}(\lambda)\right\}$ is essentially bounded.

Proof. This follows from Propositions 4.4 and 4.6.

We shall give an example which yields some of the results in N. Dunford's work. Let $H=L_{2}(0,1) \oplus L_{2}(0,1)$ and $M_{f}$ denote the multiplication operator on $L_{2}(0,1)$ for $f \in L^{\infty}(0,1)$. If

$$
A=\left[\begin{array}{ll}
M_{f} & M_{g} \\
M_{h} & M_{k}
\end{array}\right],
$$

where $f, g, h, k \in L^{\infty}(0,1)$, then clearly $A$ satisfies a second degree monic polynomial $z^{2}-N_{1} z+N_{2}$ where the coefficients

$$
N_{1}=\left[\begin{array}{ll}
M_{f+k} & 0 \\
0 & M_{f+k}
\end{array}\right]
$$

and

$$
N_{2}=\left[\begin{array}{ll}
M_{f k-g h} & 0 \\
0 & M_{f k-g h}
\end{array}\right]
$$

Thus, if we take the direct integral decomposition determined by Lebesgue measure on $[0,1]$ and $H(\lambda)=C^{2}$, then $N_{1}, N_{2}$ are obviously diagonal operators and $A$ decomposes with $A(\lambda)=\left[\begin{array}{ll}f(\lambda) & g(\lambda) \\ h(\lambda) & k(\lambda)\end{array}\right]$. Then as in Proposition 4.4, there is a Borel set $M$ so that if $g$ is the 
characteristic function on $M$, then $A$ is decomposed by $\int_{A} \oplus g(\lambda) I(\lambda) \mu d(\lambda)$ into $A_{1} \oplus A_{2}$ so that $A_{1}$ is a spectral operator of order 2 and $A_{2}$ is semi-similar to a normal operator. By Theorem $4.7, A$ is a spectral operator iff $\left\{\left\|A(\lambda)-r_{1}(\lambda)\right\|\left|r_{1}(\lambda)\right|^{-1}: \lambda \in \Lambda-M\right\}$ is essentially bounded. This later condition is equivalent (following the notation in [4]) to

$$
\frac{((f(\lambda))-k(\lambda))^{2}+g(\lambda)^{2}+h(\lambda)^{2}}{\delta(\lambda)^{2}}: \lambda \in \Lambda-M
$$

being essentially bounded where $\delta(\lambda)=\left((f(\lambda)-k(\lambda))^{2}+4 g(\lambda) h(\lambda)\right)^{1 / 2}$. Note that $\delta(\lambda)=0$ on $M$ which parallels the treatment in $[4,6$; XI]. Finally, we given an example first introduced by J. Stampfli [17] to show that sequare roots of normal operators need not be spectral. Let

$$
A_{f}=\left[\begin{array}{ll}
M_{t} & M_{f} \\
0 & M_{-t}
\end{array}\right]
$$

on $H=L_{2}(0,1) \oplus L_{2}(0,1)$ where $f \in L^{\infty}(0,1)$. Then $A_{f}^{2}$ is normal for each $f$, however $A_{f}$ is a spectral operator (in fact scalar type operator) if and only if $\left|t^{-1} f(t)\right|$ is essentially bounded. Hence, the example of J. Stampfli follows. The operator

$$
\left[\begin{array}{ll}
M_{t} & I \\
0 & M_{-t}
\end{array}\right]
$$

is the square root of a normal operator which is not a spectral operator.

We close by remarking on several areas of further research involving these methods and theorems. The theorems in $\S \S 1$ and 2 can be modified in case $\psi(z)$ takes values in certain commutative algebras of spectral operators; however, the nilpotent operators become quasinilpotent and are not necessarily of finite type. Most of the theorems can be obviously modified if the normal operators are replaced by commuting scalar type operators whenever similarity or semisimilarity is involved. Some results in this direction have been obtained and further work is in progress.

\section{REFERENCES}

1. C. Apostol, Spectral decompositions and functional calculus, Rev. Roumaine Math. Pures Appl., 13 (1968), 1481-1528.

2. E. Azoff, Spectrum and direct integral, Trans. Amer. Math. Soc., to appear.

3. H. Behncke, The structure of certain nonnormal operators. II, Indiana Univ. Math. J., 22 (1972), 301-308.

4. A. Brown, Unitary equivalence of binormal operators, Amer. J. Math., 76 (1954), 414-434. 
5. D. Deckard and C. Pearcy, On matrices over the rings of continuous complex-valued functions on a Stonian space, Proc. Amer. Math. Soc., 14 (1963), 322-328.

6. N. Dunford, A spectral theory for certain operators on a direct sum of Hilbert spaces, Math. Ann., 162 (1966), 294-330.

7. N. Dunford and J. Schwartz, Linear Operators, Parts I, II, III, Wiley Interscience (1957, 1963, 1971), New York.

8. I. Colojoara and C. Foias, Theory of Generalized Spectral Operators, Gordon and Breach (1968), New York.

9. A. N. Feldzammen, Semi-similarity invariants for spectral operators on Hilbert space, Trans. Amer. Math. Soc., 100 (1961), 277-324.

10. S. Foguel, Algebraic functions of normal operators, Israel J. Math., 6 (1968), 199-201.

11. F. Gilfeather, On a functional calculus for decomposable operators and applications to normal, operator-valud functions, Trans. Amer. Math. Soc., 176 (1973), 369-383.

12. H. Gonsher, Spectral theory for a class of nonnormal operators, Canada J. Math., 8 (1956), 449-461.

13. T. Hoover, Hyperinvariant subspaces for n-normal operators, Acta. Soc. Math., 32 (1971), 109-119.

14. B. Sz. Nagy and C. Foias, Analyse Harmonique des Operateurs de l'espace de Hilbert (1967), Budapest.

15. H. Radjavi and P. Rosenthal, Hyperinvariant subspaces for spectral and n-normal operators, Acta. Sci. Math., 32 (1971), 121-125.

16. - On roots of normal operators, J. Math. Anal. and App., 34 (1971), $653-665$.

17. J. Schwartz, $W^{*}$ Algebras, Gordon and Breach (1967), New York.

18. J. G. Stampfli, Roots of scalar operators, Proc. Amer. Math. Co., 13 (1962), 796798.

Received April 10, 1973 and in revised form January 9, 1974. This work was partially supported by the National Science Foundation under grant, NSF-31435.

UNiversity of Nebraska-Lincoln 


\section{PACIFIC JOURNAL OF MATHEMATICS}

EDITORS

RICHARD ARENS (Managing Editor)

University of California

Los Angeles, Calıfornia 90024

R. A. Beaumont

University of Washington

Seattle, Washington 98105
J. DugundJI

Department of Mathematics

University of Southern California

Los Angeles, California 90007

D. Gilbarg and J. Milgram

Stanford University

Stanford, California 94305

\section{ASSOCIATE EDITORS}
E. F. BECKENBACH
B. H. NeumanN
F. WOLF
K. YOSHIDA

\section{SUPPORTING INSTITUTIONS}

UNIVERSITY OF BRITISH COLUMBIA

CALIFORNIA INSTITUTE OF TECHNOLOGY

UNIVERSITY OF CALIFORNIA

MONTANA STATE UNIVERSITY

UNIVERSITY OF NEVADA

NEW MEXICO STATE UNIVERSITY

OREGON STATE UNIVERSITY

UNIVERSITY OF OREGON

OSAKA UNIVERSITY
UNIVERSITY OF SOUTHERN CALIFORNIA

STANFORD UNIVERSITY

UNIVERSITY OF TOKYO

UNIVERSITY OF UTAH

WASHINGTON STATE UNIVERSITY

UNIVERSITY OF WASHINGTON

AMERICAN MATHEMATICAL SOCIETY NAVAL WEAPONS CENTER 


\section{Pacific Journal of Mathematics}

\section{Vol. 55, No. $1 \quad$ September, 1974}

Robert Lee Anderson, Continuous spectra of a singular symmetric

differential operator on a Hilbert space of vector-valued functions . . . $\quad 1$

Michael James Cambern, The isometries of $L^{p}(X, K) \ldots \ldots \ldots \ldots \ldots . . \ldots$

R. H. Cameron and David Arne Storvick, Two related integrals over spaces of continuous functions ................................

Gary Theodore Chartrand and Albert David Polimeni, Ramsey theory and

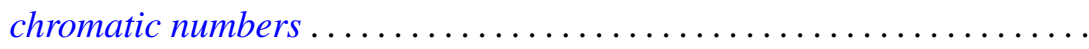

John Deryck De Pree and Harry Scott Klein, Characterization of collectively compact sets of linear operators ...................

John Deryck De Pree and Harry Scott Klein, Semi-groups and collectively compact sets of linear operators ....................... 55

George Epstein and Alfred Horn, Chain based lattices.............. 65

Paul Erdős and Ernst Gabor Straus, On the irrationality of certain series . . 85

Zdeněk Frolík, Measurable uniform spaces................... 93

Stephen Michael Gagola, Jr., Characters fully ramified over a normal

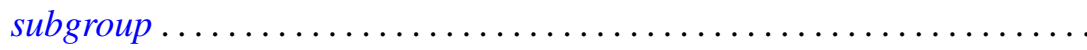

Frank Larkin Gilfeather, Operator valued roots of abelian analytic

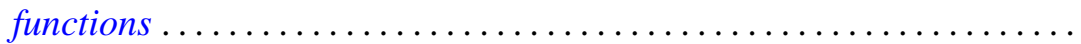

D. S. Goel, A. S. B. Holland, Cyril Nasim and B. N. Sahney, Best approximation by a saturation class of polynomial operators

James Secord Howland, Puiseux series for resonances at an embedded

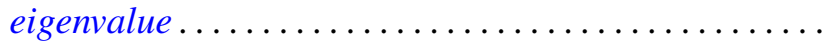

David Jacobson, Linear GCD equations .................

P. H. Karvellas, A note on compact semirings which are multiplicative

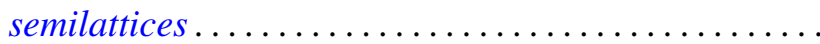

Allan Morton Krall, Stieltjes differential-boundary operators. II . .

D. G. Larman, On the inner aperture and intersections of convex sets

S. N. Mukhopadhyay, On the regularity of the $P^{n}$-integral and its application to summable trigonometric series ....... .

Dwight Webster Read, On $(J, M, m)$-extensions of Boolean algebras ....

David Francis Rearick, Multiplicativity-preserving arithmetic power series.

Indranand Sinha, Characteristic ideals in group algebras

Charles Thomas Tucker, II, Homomorphisms of Riesz spaces . . .

Kunio Yamagata, The exchange property and direct sums of indecomposable injective modules. 